\title{
Pure Geometrical Precise Orbit Determination of a LEO Based on GNSS Carrier Phase Observations
}

\section{tigg}

\section{Akbar Shabanloui, Karl Heinz Ilk}

\section{Abstract}

The interest in a precise orbit determination of Low Earth Orbiters (LEOs) especially in pure geometrical mode using GNSS observations has been rapidly grown. Conventional GNSS-based strategies rely on the GNSS observations from a terrestrial network of ground receivers (IGS network) as well as the GNSS receiver on-board LEO in double difference (DD) or in triple difference (TD) data processing modes. With the advent of precise orbit and clock products at centimetre level accuracy provided by the IGS centres, the two errors associated with broadcast orbits and clocks can be significantly reduced. Therefore, higher positioning accuracy can be expected even when only a single GNSS receiver is used in a zero difference (ZD) procedure. In this poster, the zero difference procedure has been applied to CHAMP high-low GPS SST observations then the solution has been denoted as Geometrical Precise Orbit Determination (GPOD). This pure geometrical orbit determination procedure has been declared sometimes also as "kinematical" method (e.g. G. Beutler, D. Švehla). The position accuracy of 2-3 cm of CHAMP based on high-low GPS SST carrier phase observations with zero difference procedure has been realized. These point-wise absolute positions can be used to estimate kinematical orbit of the LEOs. \begin{abstract}
GPS-SST data processing
The zero differenced of GPS-SST observations is used to estimate the geometrical absolute positions and clock offset of a LEO. The carrier phase GPS-SST observations between the GPS satellite $s$ and the LEO satellite $r$ at frequency $i$ with respect to the ambiguity parameter and all the error terms can be written as:

$$
\Phi_{r, i}^{s}(t)=\rho_{r}^{s}(t)-c d t_{r}(t)+\lambda_{i} N_{r, i}^{s}+e_{r, \Phi_{i}}^{s}(t)+\varepsilon_{\Phi_{i}}(t)
$$

here at epoch $t, \Phi_{r, i}^{s}(t)$ is the observed carrier phase between the GPS satellite $s$ and the LEO GPS receiver $r$ at frequency of $i$ in unit of length, $\rho_{r}^{s}$ is the true geometrical distance between the corresponding GPS and LEO satellites, $N_{r, i}^{s}$ is the ambiguity parameter, $\lambda_{i}$ is the wavelength of the given GPS signal at frequency of $i$ and $e_{r, i}^{s}$ is the sum of all error effects on the LEO and the GPS satellites. In the zero difference procedure, the error terms either have to be modelled with accurate specified models or have to be eliminated in the data processing procedure. The ionosphere free carrier phase observations at the epochs $t$ can be written as:

$$
\Phi_{r, 3}^{s}(t)=\rho_{r}^{s}(t)-c d t_{r}(t)+\lambda_{3} N_{r, 3}^{s}+e_{r, \Phi_{3}}^{s}(t)+\varepsilon_{\Phi_{3}}(t),
$$
\end{abstract}

with the GPS signal sending time $\tau_{r}^{s}$ and the Sagnac effect on the carrier phase observation equation as

$\Phi_{r, 3}^{s}(t)=\left\|\mathbf{R}_{Z}\left(\omega_{e} \tau_{r}^{s}\right) \mathbf{r}^{s}\left(t-\tau_{r}^{s}\right)-\mathbf{r}_{r}(t)\right\|+\lambda_{3} N_{r, 3}^{s}-c d t_{r}(t)+e_{r, \Phi_{3}}^{s}(t)+\varepsilon_{\Phi_{3}}(t)$

\section{universitätbonn}

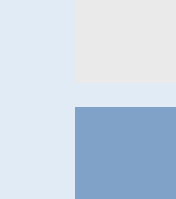

Data processing and results

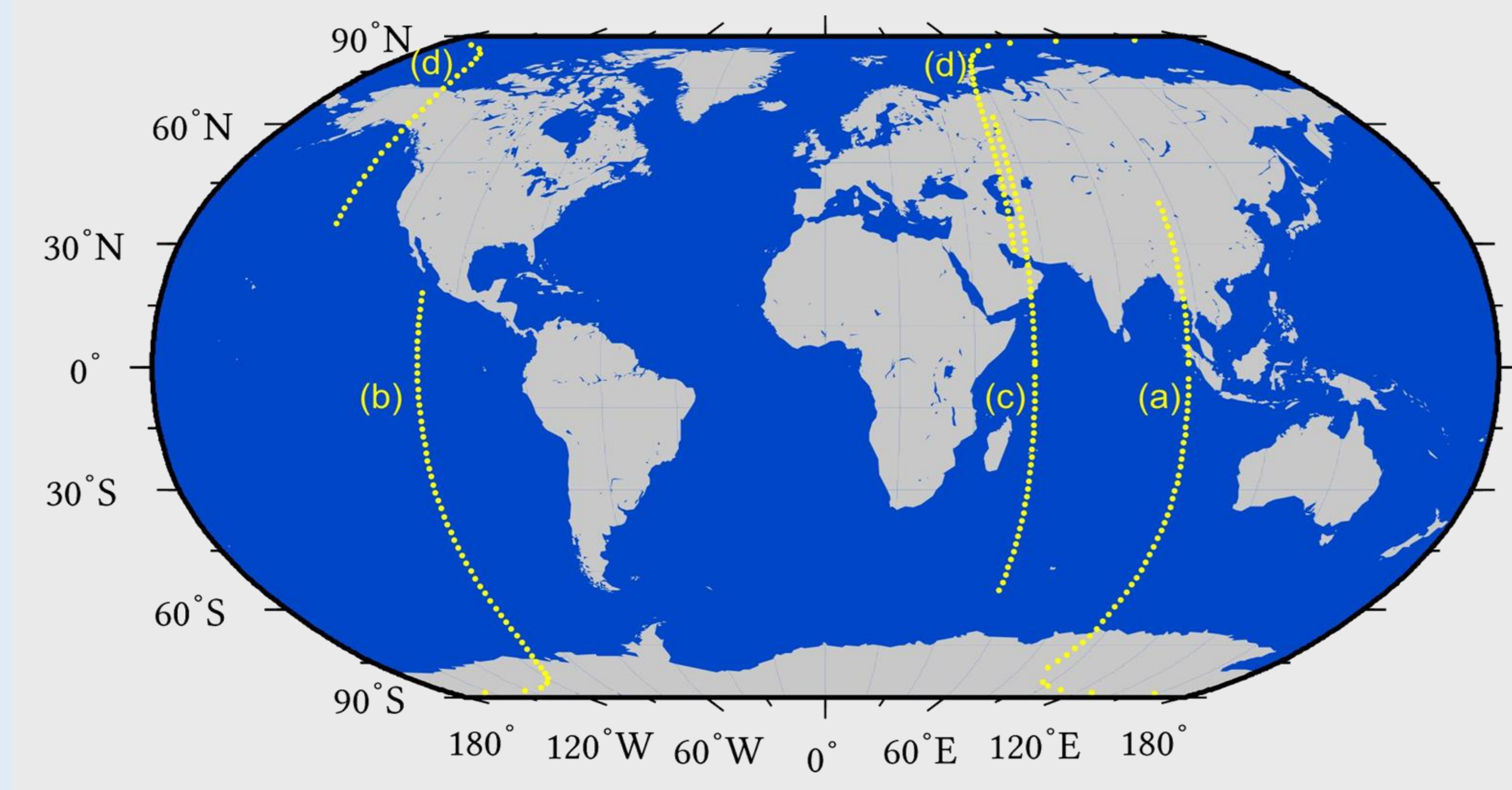

Fig. 2: The ground tracks of four 30 minutes short arcs for the time: (a) 2002/03/21 13h 30m 0.0s - 14h 00m 0.0s (b) 2002/07/20 12h 48m 0.0s - 13h $18 \mathrm{~m} 0.0 \mathrm{~s}$ (c) 2003/03/21 17h 20m 0.0s - 17h 50m 0.0s (d) 2003/03/31 17h 00m $0.0 \mathrm{~s}-17 \mathrm{~h} 30 \mathrm{~m} 0.0 \mathrm{~s}$

Fig. 3: Skyplots (elevations and azimuths) of the tracked GPS satellites applying a $15^{\circ}$ cut-off angle for a 30 minutes short arc case (b) of CHAMP. Table 1: RMS values of estimated CHAMP orbits w.r.t CHAMP PSO dynamical orbit after trend reassessing with polynomial degree five.

\begin{tabular}{|c|c|c|c|}
\hline Case & $\mathbf{X ( m )}$ & $\mathbf{Y ( m )}$ & $\mathbf{Z}(\mathbf{m})$ \\
\hline (a) & 0.0047 & 0.0096 & 0.0122 \\
\hline (b) & 0.0024 & 0.0062 & 0.0075 \\
\hline (c) & 0.0140 & 0.0171 & 0.0085 \\
\hline (d) & 0.0087 & 0.0073 & 0.0105 \\
\hline
\end{tabular}
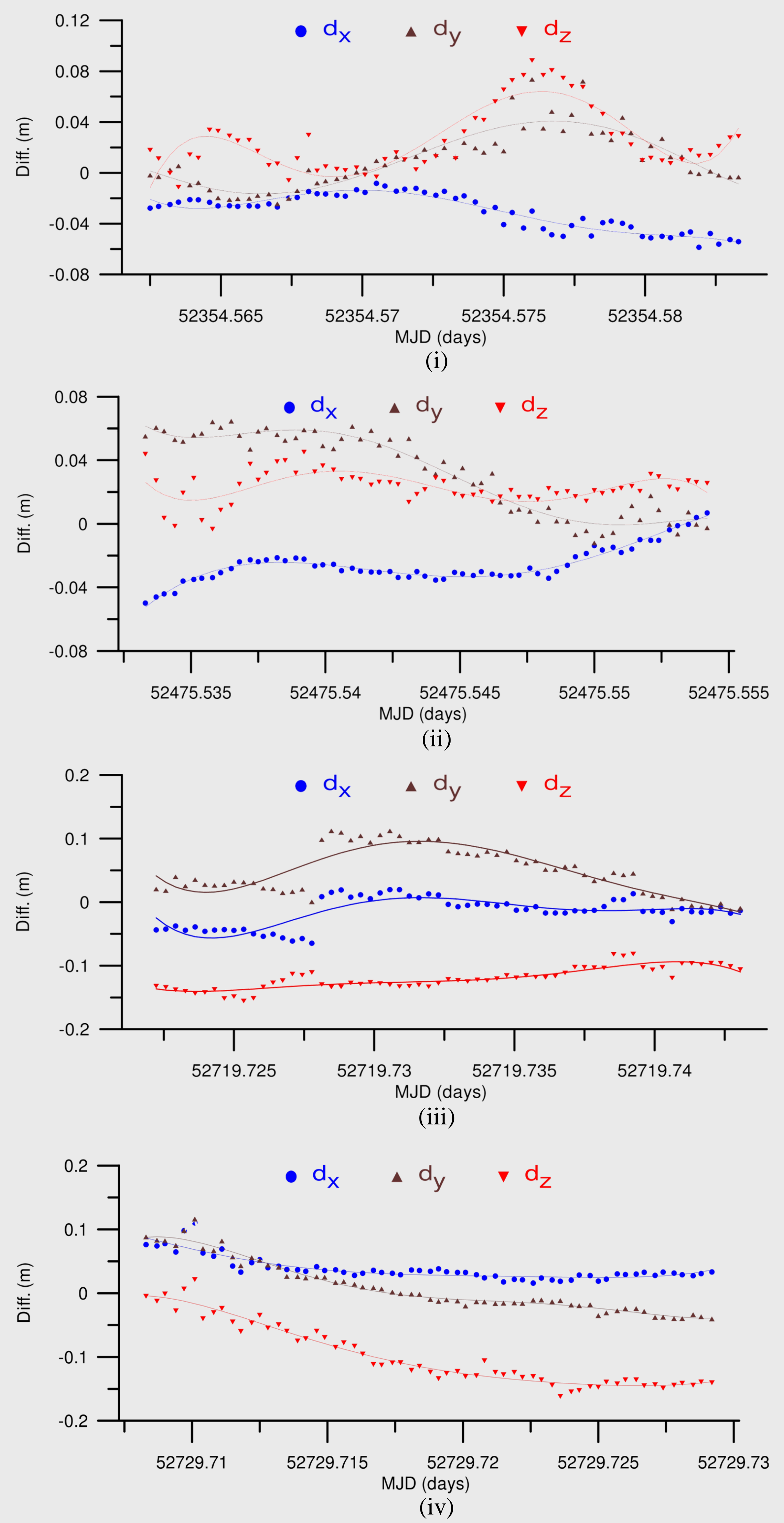

Fig. 4: Absolute position differences between estimated geometrical orbits and PSO dynamical CHAMP orbits in ITRF for the (i): case (a), (ii): case (b), (iii): case (c), (iv): case (d).

\section{Conclusions}

The proposed geometrical POD strategy can be characterized as:

- The LEO orbits are pure geometrical type, point-wise and three dimensional.

- Zero difference procedure provides an efficient possibility to estimate geometrical orbit of a LEO.
- An efficient data pre-processing and data screening procedure is very important to achieve precise geometrical LEO orbits based on GNSS SST observations.

- Real case results demonstrate that an accuracy of centimetre is achievable.
After linearization of the carrier phase observations, the GaussMarkov model can be written as,

$$
\Delta \mathbf{l}=\mathbf{A} \Delta \mathbf{x}, \quad \mathbf{W}_{\mathbf{l}},
$$

by a least square adjustment, after a few iteration the unknowns read $\Delta \hat{\mathbf{x}}_{i}=\left(\mathbf{A}_{i}{ }^{\mathrm{T}} \mathbf{W}_{\mathbf{1}} \mathbf{A}_{i}\right)^{-1} \mathbf{A}_{i}{ }^{\mathrm{T}} \mathbf{W}_{\mathbf{l}} \mathbf{l}_{i}, \mathbf{C}_{\Delta \hat{\mathbf{x}}_{i}}=\hat{\sigma}_{0}^{2}\left(\mathbf{A}_{i}{ }^{\mathrm{T}} \mathbf{W}_{\mathbf{1}} \mathbf{A}_{i}\right)^{-1}, \hat{\mathbf{x}}_{i}=\hat{\mathbf{x}}_{i-1}+\Delta \hat{\mathbf{x}}_{i}$ The estimated unknowns are the LEO absolute positions, the LEO clock offsets and the float GPS ambiguity terms at all desired epochs. To estimate GPOD of CHAMP following conditions are applied:

- Four 30 minutes short arcs of CHAMP,

• "Majority voting" techniques to data screening,

- $15^{\circ}$ cut-off angle.

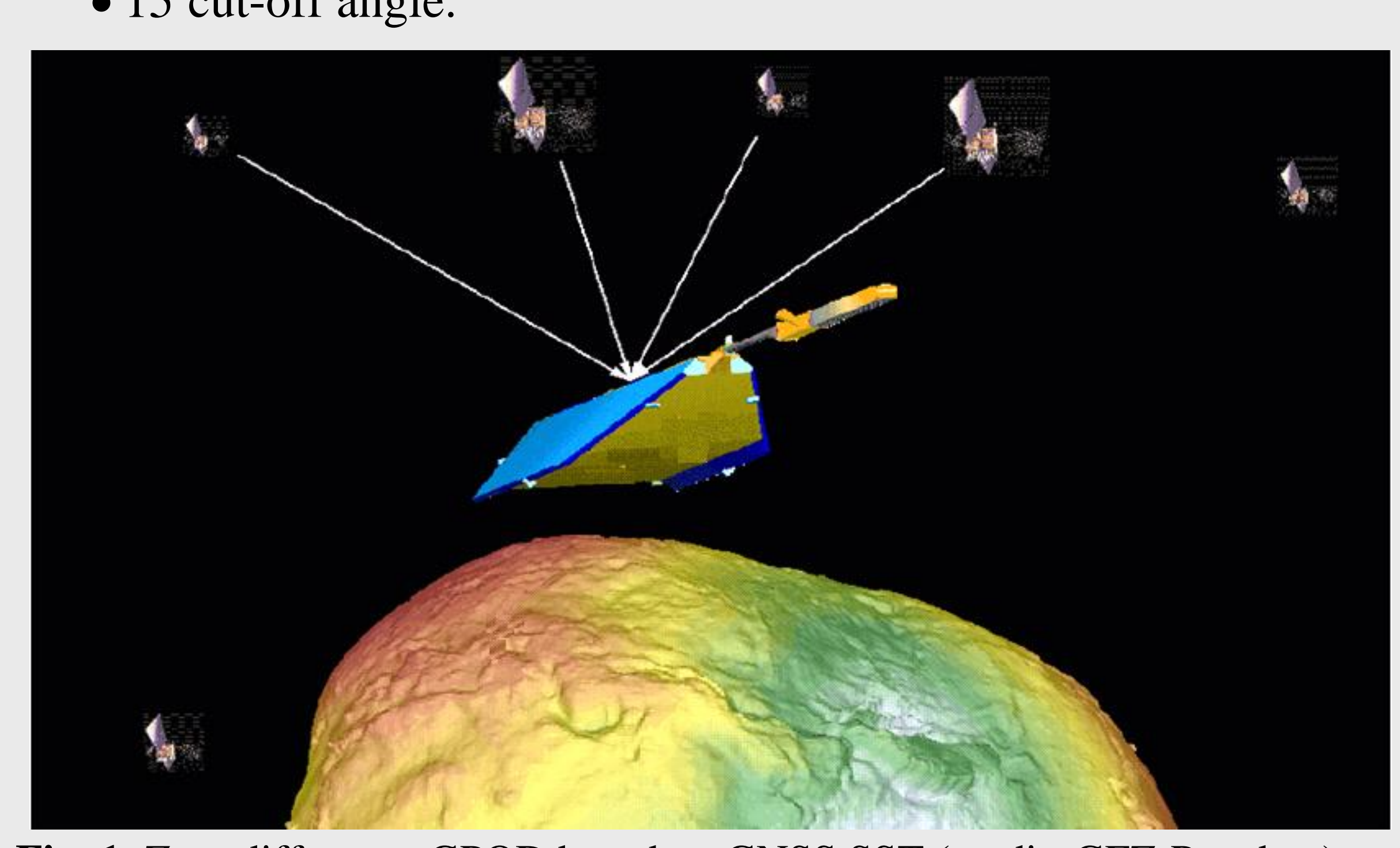

Fig. 1: Zero difference GPOD based on GNSS SST (credit: GFZ-Potsdam).
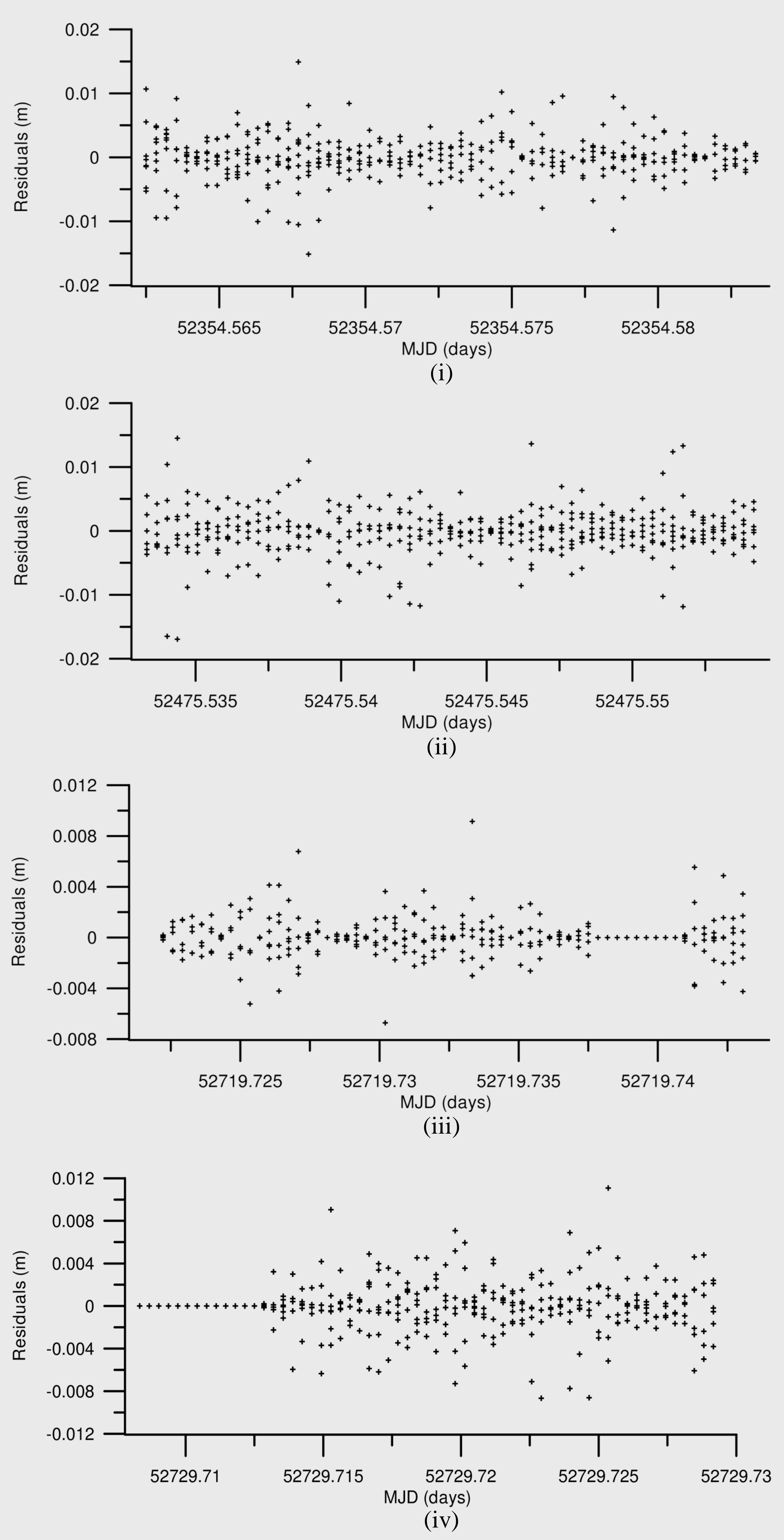

Fig. 5: Carrier phase GPS-SST observation residuals for the (i): case (a), (ii): case (b), (iii): case (c), (iv): case (d).

- The estimated geometric precise LEO orbits can be used for further investigations e.g. determination of kinematical LEO orbits for space-borne recovery of the Earth gravity field based on GNSS SST observations. 